Pacific Journal of Mathematic 


\title{
REARRANGEMENT OF SPHERICAL MODIFICATIONS
}

\author{
M. V. Mielke
}

\begin{abstract}
A "rearrangement" theorem of Wallace states essentially that if a manifold $M$ is the trace of a sequence of spherical modifications of various types then these modifications can be arranged so that the order in which they are performed is that of increasing type, their trace still being $M$. In this paper a related rearrangement problem is considered; namely, to determine bounds on how "mixed" the order of performing a sequence of modifications can be and still possess the same trace $M$.
\end{abstract}

Sections 2 through 4 are concerned with basic definitions and preliminary results. The most important of which is the establishment of an algorithm to determine a measure of how "mixed" the order of a sequence of integers is [§4]. The main results appear in $\S 5$.

2. Spherical modifications. Unless stated otherwise an $n$-manifold is a compact, differentiable $n$-dimensional manifold without boundary.

Let $V_{1}$ be an $n$-manifold and suppose $S^{i}$ is an $i$-sphere homeomorphically and smoothly imbedded in $V_{1}$ with a trivial normal bundle. Then $S^{i}$ has a neighborhood of the form $S^{i} \times D^{n-i}$. $\left(D^{n-i}\right.$ is an $(n-i)$ - disc). Clearly the boundary of $S^{i} \times D^{n-i}=S^{i} \times S^{n-i-1}=$ the boundary of $D^{i+1} \times S^{n-i-1}$. Smoothly identifying the boundary of $D^{i+1} \times S^{n-i-1}$ with the boundary of $\left(V_{1}\right.$-interior $\left.\left(S^{i} \times D^{n-i}\right)\right)$ results in a new manifold $V_{2}, V_{2}$ is said to be obtained from $V_{1}$ by a spherical modification of type $i$, or by an $i$-type modification. ([7], p. 504).

Associated to the spherical modification is an $n+1$-manifold $W$ called the trace of the modification. The boundary of $W=V_{1} \cup V_{2}$ and the triple ( $\left.W ; V_{1}, V_{2}\right)$ is a manifold triad in the sense of [4] page 2. As a matter of convention, performing a type-1 modification on $V_{1}$ is taken to mean $V_{1}=\varnothing, V_{2}=$ an $n$-sphere, and the trace is an $n+1$ - disc. The $n$ will be clear from context. For a further discussion of the trace see [8] page 775 .

\section{Realizable sequences.}

DEFINITION 3.1. An admissible sequence $S(n)$ is a finite sequence of integers $a_{1}, a_{2}, \cdots, a_{l}$ with $a_{1}=-1, a_{l}=n-1,-1 \leqq a_{i} \leqq n-1$ for $i=1,2, \cdots, l$ and $a_{i} \neq a_{j}$ if $i \neq j$.

Definition 3.2. Let $S(n)$ be an admissible sequence. $S(n)$ is re- 
alizable by an $n$-manifold $M$ if $M$ is diffeomorphic to the combined trace of a sequence of spherical modifications of type $a_{1}, a_{2}, \cdots, a_{l}$. More explicitly, there exists a sequence of $(n-1)$-manifolds $V_{1}, V_{2}$, $\cdots, V_{l+1}$ where $V_{i+1}$ can be obtained from $V_{i}$ by performing a finite number of type $a_{i}$ modifications on $V_{i}$ and the combined trace of these modifications is diffeomorphic to $M$.

Note that $S(n)$ only tells the type of modifications that appear and gives no information on how many modifications of the various types occur. Also it is only necessary to consider admissible sequences since $M$ is assumed to be without boundary and compact.

When no confusion will arise the $n$ in $S(n)$ will be deleted.

The following lemmas will be used in proving the results of $\S 5$.

LEMMA 3.1. Let $S=\left\{a_{1}, \cdots, a_{i}, a_{i+1}, \cdots, a_{j}, \cdots, a_{l}\right\}$ be an admissible sequence realizable by $M$. If all the integers following $a_{i}$ and preceding $a_{j}$ in $S$ are larger than $a_{j}$ then the sequence $S^{\prime}=\left\{a_{1}, \cdots\right.$, $\left.a_{i}, a_{j}, a_{i+1}, \cdots, a_{l}\right\}$ is also realizable by $M$.

\section{Proof. See $\S 4$ of [7] or $\S 4$ of [4].}

LEMMA 3.2. Let $S=\left\{a_{1}, \cdots, a_{i}, a_{i+1}, \cdots, a_{i+k}, \cdots, a_{l}\right\}$ be an admissible sequence realizable by $M$. If $a_{i+j}>a_{i+j+1}$ for $j=0,1, \cdots, k-1$ then $M$ is diffeomorphic to the trace of a sequence of modifications with all modifications of type $a_{i+j}(j=0,1, \cdots, k)$ performed on a single manifold. More explicitly, there exists a sequence of manifolds $V_{1}, \cdots, V_{i}, V_{i+k+1}, V_{i+k+2}, \cdots, V_{l+1}$ where $V_{i+k+1}$ can be obtained from $V_{i}$ by modifications of type $a_{i+j}(j=0,1, \cdots, k)$ (the $a_{i+j}$-spheres $j=0,1, \cdots, k$ determining these modifications can be assumed mutually disjointly imbedded in $V_{i}$ ). Further $V_{j+1}$ can be obtained from $V_{j}$ by $a_{j}$ type modifications for $i \neq j \neq i+k$ and the combined trace of all the modifications is diffeomorphic to $M$.

\section{Proof. See $\S 4$ of [4], $\S 4$ of [7], or [9].}

THEOREM 3.1. (Rearrangement Theorem). Let $S$ be an admissible sequence realizable by $M$. Then the sequence $S^{\prime}=\left\{a_{1}, \cdots, a_{l}\right\}$ composed of the same integers as $S$ and satisfying $a_{i}<a_{i+1}$ for $i=1,2$, $\cdots, l-1$ is also realizable by $M$.

Proof. This follows easily by repeated application of Lemma 3.1. Theorem 3.1 also follows from a more general rearrangement theorem due to Smale [6] and Wallace [7]. Also see ([4], p. 44, Th. 4.8). The above theorem asserts that any sequence of modifications 
realizable by $M$ can still be realized by $M$ when those modifications are performed in order of increasing type. The purpose of this paper is to study how "mixed" the order of the modifications may be and still be realizable by $M$. To this end a study of sequences will now be made.

\section{Shifts on sequences.}

Definition 4.1. Let $S=\left\{a_{i}\right\} i=1,2, \cdots, l$ be a finite sequence of distinct integers. A shift of $S$ consists of removing some $a_{j}$ from $S$ and replacing it in $S-\left\{a_{j}\right\}$ in a position different from its original position.

Definition 4.2. $d(S)=$ minimum number of shifts needed to put $S$ in natural order.

A method of computing $d(S)$ which will be useful in proving the main results of this paper will now be described.

Definition 4.3. The full decreasing subsequence $S_{1}=\left\{a_{1 j}\right\} j=1$, $\cdots, n_{1}$ of $S$ is the subsequence of $S$ obtained as follows: $a_{11}$ is the first integer appearing in $S, a_{12}$ is the first integer following $a_{11}$ in $S$ satisfying $a_{12}<a_{11}, a_{13}$ is the first integer following $a_{12}$ in $S$ satisfying $a_{13}<a_{12}$ etc. After a finite number of such steps the process must terminate with the last element so determined being $a_{1 n_{1}}$.

DeFinition 4.4. The number of decreasing subsequences of $S$ is the smallest integer $m$ satisfying $\left(S-\bigcup_{i=1}^{m} S_{i}\right)=\varnothing$ where $S_{i}=\left\{a_{i j}\right\}$ $j=1, \cdots, n_{i}$ is the full decreasing subsequence of $\left(S-\bigcup_{j=1}^{i-1} S_{j}\right)=S$ with the subsequences $S_{1}, S_{2}, \cdots, S_{i-1}$ deleted.

TheOREM 4.1. Let $S$ be a sequence composed of $l$ distinct integers. If $S$ has $m$ decreasing subsequences then $d(S)=l-m$.

Proof. The proof will be by induction on the length $l$ of $S$. If $l=1$ the theorem clearly holds.

Assume now that the theorem is valid for sequences of length $<l$. Let $S$ have length $l$ and $m$ decreasing subsequences with $n_{i}$ integers in the $i^{t h}$ subsequence $S_{i}$.

If $d(S)=0$ it is clear that $n_{i}=1$ for $i=1, \cdots, m$. Thus $l=m$ and $d(S)=l-m$.

Suppose then that $d(S) \neq 0$. Under this assumption there is defined a unique integer $p$ satisfying $n_{p}>1$ and if $p<r \leqq m$ then $n_{r}=1$. Let $S^{\prime}$ be $S$ with $a_{p n_{p}}$ deleted if $p=m$ or if $p \neq m$ and 
$a_{p 1}<a_{p+1,1}$, and with $a_{p 1}$ deleted if $p \neq m$ and $a_{p+1,1}<a_{p 1}$.

LEMma 4.1. $d(S) \leqq d\left(S^{\prime}\right)+1$.

Proof. One can imagine the $d\left(S^{\prime}\right)$ shifts used on $S^{\prime}$ to put it in natural order as being performed on $S$ (simply ignore $a_{p 1}$ (or $a_{p n_{p}}$ )). Hence after the $d\left(S^{\prime}\right)$ shifts on $S$ one obtains a sequence $S^{\prime \prime}$ in which all integers are in natural order except for possibly one, $a_{p 1}$ (or $\left.a_{p n_{p}}\right)$. At most, then, one more shift will put $S^{\prime \prime}$ in natural order. This proves the lemma.

\section{Lemma $4.2 . \quad d\left(S^{\prime}\right)=l-1-m$.}

Proof. Since the length of $S^{\prime}$ is $l-1$ the induction hypothesis implies $d\left(S^{\prime}\right)=l-1-m^{\prime}$ where $m^{\prime}=$ number of decreasing subsequences of $S^{\prime}$. It must be shown that $m=m^{\prime}$.

Let $A=\left\{S_{1}, \cdots, S_{m}\right\}$ and $A^{\prime}=\left\{S_{1}^{\prime}, \cdots, S_{m^{\prime}}^{\prime}\right\}$ be the sets of full decreasing subsequences of $S$ and $S^{\prime}$ respectively. Clearly deleting an element from the $p^{\text {th }}$ sequence $S_{p}$ does not change any of the preceding sequences. Hence $A$ and $A^{\prime}$ are identical up to the $p^{\text {th }}$ sequence. Consider now the $p^{\text {th }}$ sequence.

Case 1. $p=m$ or if $p \neq m$ then $a_{p 1}<a_{p+1,1}$. This implies that $a_{p 1}$ is not deleted and thus sequence $p$ of both $A$ and $A^{\prime}$ begins with $a_{p 1}$. It will now be shown that $S_{i}=S_{i}^{\prime}$ for $p<i \leqq m$. For suppose that some $a_{i 1}(i>p)$ appears in $S_{p}^{\prime}$. The existence of such an $a_{i 1}$ implies that $p \neq m$, thus $a_{p 1}<a_{p+1,1}$. By construction $a_{p+1,1}<a_{p+2,1}<$ $\cdots<a_{m 1}$ and consequently $a_{p 1}<a_{i 1}$. But also by construction $a_{p 1}$ is the largest integer of $S_{p}^{\prime}$, hence no $a_{i 1}(p<i \leqq m)$ can appear in $S_{p}^{\prime}$. From the relation $a_{i 1}<a_{i+1,1}(i=p+1, \cdots, m-1)$ it easily follows now that each $a_{i 1}(p<i \leqq m)$ constitutes a sequence of $A^{\prime}$. Therefore $m=m^{\prime}$.

Case 2. $p \neq m$ and $a_{p+1,1}<a_{p 1}$. This implies that $a_{p 1}$ is deleted. Suppose now that $a_{i 1}(i>p)$ appears in $S_{p}^{\prime}$. By definition, $a_{i 1}$ appears in $S$ after $a_{p 1}$. Let $a_{p j}$ be the smallest integer in $S_{p}$ that precedes $a_{i 1}$ in $S$. Then $a_{p j}<a_{i 1}$ or $a_{i 1}$ would be in $S_{p}$. Furthermore $a_{p j}$ cannot appear in $S_{p}^{\prime}$, since $a_{i 1}$ does. But the only way $a_{p j}$ could fail to be in that sequence is by being deleted; that is, $j=1$. However since $a_{p+1,1}<a_{p 1}$ and $a_{p+1,1}$ follows $a_{p 1}$ in $S$ there must be an element of the form $a_{p k}(k \neq 1)$ preceding $a_{p+1,1}$. Since $a_{i 1}$ is $a_{p+1,1}$ or follows it in $S$, $a_{i 1}$ must also follow $a_{p k}(k \neq 1)$. Hence $a_{p j} \neq a_{p 1}$ which is a contradiction.

The same argument as in Case 1 now shows that each $a_{i 1}$ $(p<i \leqq m)$ constitutes a sequence of $A^{\prime}$. Hence $m=m^{\prime}$.

In all cases, then, $m=m^{\prime}$ which proves the lemma. 
LEMMA 4.3. $d(S) \geqq l-m$.

Proof. Consider $S_{i}=\left\{a_{i j}\right\} j=1, \cdots, n_{i}$. These elements appear in $S$ in the given order. By construction $a_{i n_{i}}<\cdots<a_{i 2}<a_{i 1}$, hence it clearly takes at least $n_{i}-1$ shifts to put $S_{i}$ in natural order. Further any shift involving an element of $S_{i}$ does not affect the relative order of the elements of $S_{j}(i \neq j)$. Thus the number of shifts needed is $\geqq \sum_{i=1}^{m}\left(n_{i}-1\right)=\left(\sum_{i=1}^{m} n_{i}\right)-m=l-m$ and the lemma is proved.

Combining Lemma 4.1 and 4.2 gives $d(S) \leqq l-m$ and in view of Lemma $4.3 d(S)=l-m$. This completes the proof of Theorem 4.1.

REMARK. It is clear that the "natural order" of $S$ is not essential to the above arguments and the results of this section can be generalized to the situation of finding the number of shifts needed to put $S$ in a given order, not necessarily the natural order. Theorem 4.1 still holds in this broader sense and $d$ becomes a distance function on the set of all orderings of $S$.

5. The main result. Denote by $C(M)$ the strong category of $M(\mathrm{cf}[1])$.

THEOREM 5.1. Let $S$ be an admissible sequence of length $l$. If $S$ is realizable by an $n$-manifold $M(n>0)$ then $2 \leqq d(S)+C(M) \leqq$ $l \leqq n+1$.

Proof. Since $M$ is an $n$-manifold the only modifications that can occur are of type $-1,0,1, \cdots, n-1$, thus $l \leqq n+1$. A simple cohomological argument shows $C(M) \geqq 2$ and thus

$$
2 \leqq C(M) \leqq d(S)+C(M) \text {. }
$$

It remains then to establish $d(S)+C(M) \leqq l$, which is equivalent to $C(M) \leqq l-d(S)$. In view of Theorem 4.1, then, Theorem 5.1 will follow if it is shown that $C(M) \leqq m$.

LEMmA 5.1. If $S$ is an admissible sequence realizable by $M$ then $C(M) \leqq m$ where $m$ is the number of decreasing subsequences of $S$.

Proof. Let $A=\left\{S_{1}, S_{2}, \cdots, S_{m}\right\}$ be the set of decreasing subsequences of $S$. Since $S$ is admissible $n_{1}=n_{m}=1, a_{11}=-1$ and $a_{m 1}=$ $n-1$. Let $S^{\prime}$ be the sequence given in $A$; that is,

$$
S^{\prime}=a_{11}, a_{21}, a_{22}, \cdots, a_{2 n_{2}}, \cdots, a_{m-1,1}, \cdots, a_{m-1, n_{m-1}}, a_{m 1} .
$$

It will first be shown that $S^{\prime}$ is realizable by $M$. Since $a_{11}=-1$ 
it follows that all admissible sequences composed of the same integers as $S$ begin with $a_{11}$. By definition all the integers between $a_{2 i}$ and $a_{2, i+1}$ in $S$ are larger than $a_{2, i+1}$ for $i=1,2, \cdots, n_{2}$. Thus by repeated application of Lemma 3.1 the sequence $S_{1}^{\prime}=a_{11}, a_{21}, a_{22}, \cdots, a_{2 n_{2}}, \cdots$ is realizable by $M$ where the last $\ldots$ represent the sequence $S$ with $a_{11}, a_{2 i}\left(i=1,2, \cdots, n_{2}\right)$ deleted. Repeating the argument using sequences $j(j=3, \cdots, m)$ of $A$ one easily obtains the result.

Now by repeated application of Lemma 3.2 there exists a sequence of $(n-1)$-manifolds $V_{1}, V_{2}, \cdots, V_{m+1}$ where $V_{i+1}$ can be obtained from $V_{i}$ by modifications of type $\alpha_{i j}\left(j=1,2, \cdots, n_{i}\right)$ and the combined trace of all these modifications is diffeomorphic to $M$. A detailed study of the trace will now be made.

Since $M$ is connected the number of -1 type modifications needed can always be assumed to be 1 ([7], p. 518). Thus

$$
V_{1}=\varnothing, V_{2}=S^{n-1}
$$

and the trace $T_{1}$ of this modifications is an $n$-dise $D_{1}$ with $\partial D_{1}=V_{2}=$ $S^{n-1}$ (" $\partial$ " denotes boundary). $V_{3}$ is obtained from $V_{2}$ by $a_{2 i}(i=1,2$, $\left.\cdots, n_{2}\right)$ type modifications. Denote by $N\left(a_{2 i}\right)$ the number of $a_{2 i}$ type modifications involved. Then $N_{2}=\sum_{i=1}^{n ?} N\left(a_{2 i}\right)$ is the number of modifications used in going from $V_{2}$ to $V_{3}$. Each $a_{2 i}$ type modification is determined by an $a_{2 i}$-sphere imbedded in $V_{2}$ possessing a normal neighborhood of the form $S^{a 2 i} \times D^{n-a 2 i-1}$. Thus there is imbedded in $V_{2} N_{2}$ such spheres having mutually disjoint trivial normal neighborhoods.

Let $T_{2}$ be the trace of these $N_{2}$ modifications. Then $T_{1} \cup T_{2}$ is homeomorphic to the topological space $X$ constructed as follows: Any $n$-disc $D^{n}$ is of the form $D^{\jmath} \times D^{n-j}$, thus $\partial D^{n}=S^{\jmath-1} \times D^{n-j} \cup D^{j} \times S^{n-\jmath-1}$. Attach $N_{2}$ different $n$-discs to $V_{2}$ by identifying $S^{\alpha i} \times D^{n-a i-1} \subset \partial D^{n}$ (let $j=a_{i}+1$ ) with the normal neighborhood of the $a_{i}$-sphere, the attaching diffeomorphisms being determined by the spherical modifications involved. Thus $X$ is $D_{1}$ with $N_{2} n$-dises attached to $\partial D_{1}$. Hence $T_{1} \cup T_{2}=D_{1} \cup D_{2}$ where $D_{1}$ is an $n$-dise and $D_{2}$ is the union of $N_{2}$ mutually disjoint $n$-discs. For a detailed discussion of this description of the trace see $\S 3$ of either [5] or [8].

Repeating this argument through $V_{m+1}$ one has that $M=\bigcup_{i=1}^{m}$ $T_{i}=\bigcup_{i=1}^{m} D_{i}$ where $D_{i}$ is the union of $N_{i}=\sum_{j=1}^{n_{i}} N\left(a_{i j}\right)$ mutually disjoint $n$-discs. If $\left(C_{1}, C_{2}, \cdots, C_{k}\right)$ is a set of mutually disjoint $n$-dises in a connected $n$-manifold (which $M$ is) then $C_{1}$ can be joined to $C_{2}$ by a smooth arc $\alpha$ so that $\alpha \cap \bigcup_{i=1}^{k} C_{i}=$ two points, one in $\partial C_{1}$ and one in $\partial C_{2}$. Thus $C_{1}$ and $C_{2}$ can be joined to form a set $E_{2}$ which is contractible in itself. Repeating this construction on $\left(E_{2}, C_{3}, \cdots, C_{l_{k}}\right)$ starting with $E_{2}$ and $C_{3}$ to form $E_{3}$ one obtains a set $E_{k}$ which is contractible in itself. 
Hence $M$ can be covered by $m$ such contractible sets and if each of them is slightly "blown up" then $M$ can be covered by $m$ contractible in themselves, open sets. Thus $C(M) \leqq m$. This completes the proof of Lemma 5.1 and thus of Theorem 5.1.

CoROLlary 5.1. If an admissible sequence $S$ of length $l$ is realizable by $M$ and $l=C(M)$ then $d(S)=0$. In particular if $C(M)=$ $n+1$ then any admissible sequence realizable by $M$ must satisfy $d(S)=0$.

Proof. The corollary follows directly from Theorem 5.1.

There are many manifolds $M$ satisfying $C(M)=n+1$. For example $C\left(P^{n}\right)=n+1$ where $P^{n}$ is $n$-dimensional real projective space. If $n$ is even, all connected manifolds $M$ in the cobordism class of $P^{n}$ satisfy $C(M)=n+1$. Actually it can be shown that every cobordism class contains manifolds satisfying this condition and for even dimensional manifolds at least half of all cobordism classes contain only manifolds $M$ satisfying $C(M)=n+1$ if $M$ is connected [3].

If $l$ is the length of an admissible sequence $S$ realizable by $M$ then Theorem 5.1 asserts that $d(S)+C(M) \leqq l$ and since $C^{\prime}(M) \leqq$ $C(M)\left(C^{\prime}(M)\right.$ is the category of $\left.M\right)$ it follows that $d(S)+C^{\prime}(M) \leqq l$. One question that arises is when can an $S$ be found so that equality holds, i.e., $d(S)+C(M)=l$. A connection between this question and the Poincare conjecture will now be given.

CoROLlary 5.2. Let $M$ be an n-manifold which is a homotopy sphere. If there exists an admissible sequence $S$ of length $l$ realizable by $M$ satisfying $d(S)+C^{\prime}(M)=l$ then $M$ is homeomorphic to the $n$-sphere $S^{n}$.

Proof. Since $M$ is a locally contractible, compact, finite dimensional metric space it is an absolute neighborhood retract ([2], p. 32). It is assumed that the homotopy groups vanish in all positive dimensions $\leqq n-1$ thus by Theorem 18.2 of [1] $C^{\prime}(M) \leqq 2$ and consequently $C^{\prime}(M)=2$. Therefore $d(S)=l-2 . \quad d(S)=l-m$ implies then that $m=2$. But $l=\sum_{i=1}^{m} n_{i}=\sum_{i=1}^{2} n_{i}=n_{1}+n_{2}=2$ (recall that $n_{1}=$ $\left.n_{m}=1\right)$; that is, $S$ is the sequence $(-1, n-1)$, and since $M$ is connected it can be assumed that there is only one modification of each type ([7], Th. 4). In terms of functions this means there exists a Morse function on $M$ with exactly two nondegenerate critical points. (cf. [4], p. 30) Applying Reeb's theorem ([5], p. 25) one has that $M$ is homeomorphic to $S^{n}$. This completes the proof. 


\section{REFERENCES}

1. R. H. Fox, On the Lusternik-Schnirelman category, Ann. of Math. 42 (1941), 333370 .

2. S. T. Hu, Homotopy theory, Academic Press, New York, 1959.

3. M. V. Mielke, Spherical modifications and coverings by cells, (to appear in Duke Math. J.) March. 1969.

4. J. W. Milnor, Lectures on the h-cobordism theorem, Princeton Mathematical Notes, 1965.

5. - Morse theory, Annals of Math, Studies No. 51, Princeton, 1963.

6. S. Smale, On gradient dynamical systems, Ann. of. Math. 74 (1961), 199-206.

7. A. H. Wallace, Modifications and cobounding manifolds, Canad. J. Math. 12 (1960) 503-528.

8. - Modifications and cobounding manifolds II, J. Math. Mech. 10 (1961), 773-809.

9. A geometric method in differential topology, Bull. Amer. Math. Soc. 68 (1962), 533-542.

Received July 20, $196 \pi$.

University of Miami

Coral Gables, Florida 


\section{PACIFIC JOURNAL OF MATHEMATICS}

\section{EDITORS}

H. ROYDEN

Stanford University

Stanford, California

R. R Phelps

University of Washington

Seattle, Washington 98105
J. DUGUNDJI

Department of Mathematics

University of Southern California

Los Angeles, California 90007

RICHARD ARENS

University of California

Los Angeles, California 90024

\section{ASSOCIATE EDITORS}
E. F. BECKENBACH
B. H. NeumanN
F. WOLF
K. YosidA

\section{SUPPORTING INSTITUTIONS}

UNIVERSITY OF BRITISH COLUMBIA

CALIFORNIA INSTITUTE OF TECHNOLOGY

UNIVERSITY OF CALIFORNIA

MONTANA STATE UNIVERSITY

UNIVERSITY OF NEVADA

NEW MEXICO STATE UNIVERSITY

OREGON STATE UNIVERSITY

UNIVERSITY OF OREGON

OSAKA UNIVERSITY

UNIVERSITY OF SOUTHERN CALIFORNIA
STANFORD UNIVERSITY

UNIVERSITY OF TOKYO

UNIVERSITY OF UTAH

WASHINGTON STATE UNIVERSITY

UNIVERSITY OF WASHINGTON

AMERICAN MATHEMATICAL SOCIETY CHEVRON RESEARCH CORPORATION TRW SYSTEMS

NAVAL WEAPONS CENTER 


\section{Pacific Journal of Mathematics

Vol. 28, No. $1 \quad$ March, 1969

Patrick Robert Ahern, On the geometry of the unit ball in the space of real annihilating measures .............................. 1

Kirby Alan Baker, Equational classes of modular lattices ............. 9

E. F. Beckenbach and Gerald Andrew Hutchison, Meromorphic minimal surfaces ......................................... 17

Tae Ho Choe, Intrinsic topologies in a topological lattice ..............

John Bligh Conway, A theorem on sequential convergence of measures and

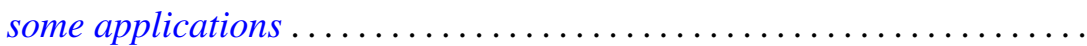

Roger Cuppens, On the decomposition of infinitely divisible probability laws without normal factor.

Lynn Harry Erbe, Nonoscillatory solutions of second order nonlinear

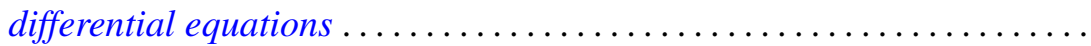

Burton I. Fein, The Schur index for projective representations of finite

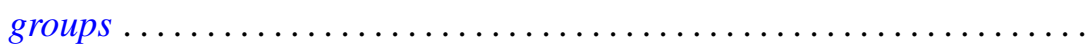

Stanley P. Gudder, A note on proposition observables............... 101

Kenneth Kapp, On Croisot's theory of decompositions ............... 105

Robert P. Kaufman, Gap series and an example to Malliavin's theorem . . . 117

E. J. McShane, Robert Breckenridge Warfield, Jr. and V. M. Warfield,

Invariant extensions of linear functionals, with applications to measures and stochastic processes ................

Marvin Victor Mielke, Rearrangement of spherical modifications ...

Akio Osada, On unicity of capacity functions ..............

Donald Steven Passman, Some 5/2 transitive permutation groups ...

Harold L. Peterson, Jr., Regular and irregular measures on groups and dyadic spaces...

Habib Salehi, On interpolation of $q$-variate stationary stochastic processes...

Michael Samuel Skaff, Vector valued Orlicz spaces generalized

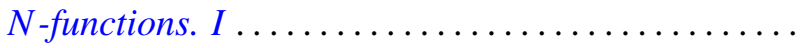

A. J. Ward, On $H$-equivalence of uniformities. II...........

Thomas Paul Whaley, Algebras satisfying the descending chain condition for subalgebras...

G. K. White, On subgroups of fixed index

Martin Michael Zuckerman, A unifying condition for implications among the axioms of choice for finite sets ................. 